J. Phys. IV France 138 (2006) 163-169

(C) EDP Sciences, Les Ulis

DOI: 10.1051/jp4:2006138019

\title{
Caractérisation du seuil de dommage dans les cristaux de saphir dopé au titane avec des impulsions de durée nanoseconde, picoseconde et femtoseconde
}

\author{
F. Canova ${ }^{1}$, J.-P. Chambaret ${ }^{1}$, G. Mourou ${ }^{1}$, M. Sentis ${ }^{2}$, O. Uteza ${ }^{2}$, \\ P. Delaporte ${ }^{2}$ et T. Itina ${ }^{2}$ \\ ${ }^{1}$ LOA, ENSTA, École Polytechnique, Chemin de la Hunière, 91761 Palaiseau Cedex, France \\ ${ }^{2}$ LP3 Pôle Scientifique et Technologique de Luminy, 163 avenue de Luminy, \\ C. 917, 13288 Marseille Cedex 9, France
}

Résumé. Le verrou principal pour le développement des systèmes femtoseconde robustes et rentables est l'incertitude du seuil de dommages du cristal de Ti : Saphir. Nous avons d'abord caractérisé le seuil diélectrique de dommages du Ti : Saphir.

\section{INTRODUCTION ET PROBLÉMATIQUE}

L'apparition du saphir dopé au titane (Ti : Saphir) dans le développement des lasers femtosecondes solides ultra intenses à amplification par dérive de fréquence (CPA), a permis une évolution sans précédent de la progression vers les très hautes puissances crête et très hautes intensités. On atteint aujourd'hui des puissances crête de l'ordre du Pétawatt $\left(10^{15} \mathrm{~W}\right)$ à faible cadence et des centaines de Terawatt à haute cadence $(>10 \mathrm{~Hz})$, et ceci dans des laboratoires de dimensions raisonnables. Ces sources, maintenant commercialisées (jusqu'à $100 \mathrm{TW}$ ), ont généré une véritable révolution dans le domaine de la recherche fondamentale en physique des très hautes intensités et des plasmas: Accélération de particules, sources X et XUV cohérentes, etc., ainsi que dans le domaine médical (proton thérapie pour le traitement de tumeurs cancéreuses) ou environnemental pour la transmutation de radio isotopes (retraitement des déchets radioactifs). Pour accéder à ces futures applications, les sources laser de très haute intensité fonctionnant à haute cadence, doivent encore franchir un certain nombre de verrous afin d'améliorer leur niveau de performances et d'assurer un fonctionnement plus fiable, plus reproductible et à moindre coût.

Une des difficultés majeures réside dans la contrainte d'utilisation de cristaux amplificateurs de plus en plus grands et coûteux. Des monocristaux de saphir dopé au titane de plus de $100 \mathrm{~mm}$ de diamètre sont maintenant accessibles sur le marché mais à des coûts élevés (80 000 Euros) et avec des délais d'approvisionnement importants (un an environ). Ils sont « pompés optiquement » par des lasers Néodyme YAG doublés en fréquence $(532 \mathrm{~nm})$ à des fluences proches du seuil d'endommagement du matériau, lui-même estimé entre 5 et $10 \mathrm{~J} / \mathrm{cm}^{2}$ (impulsions nanoseconde). Ces sources de pompage étant partiellement cohérentes, il existe des surintensités ponctuelles de durée caractéristique picoseconde, résultant du battement entre les modes du laser. Ce régime de fonctionnement de pompage (1 à $4 \mathrm{~J} / \mathrm{cm}^{2}$ ) est nécessaire pour obtenir un gain raisonnable dans les étages amplificateurs de puissance, la fluence de saturation du Saphir dopé au titane étant de l'ordre de $1 \mathrm{~J} / \mathrm{cm}^{2}$. Pour accéder à des modes de fonctionnement reproductibles, récurrents et sans risques de dommage pour les matériaux amplificateurs, les différents laboratoires et les industriels des lasers engagés dans ces développements utilisent des fluences de pompage conservatives de l'ordre $\mathrm{du} \mathrm{J} / \mathrm{cm}^{2}$ voire même inférieures. Les efficacités d'extraction sont alors relativement modestes (35 à $40 \%$ de l'énergie de pompe) alors que la limite théorique est de $65 \%$ ! 
L'étude de l'endommagement des cristaux utilisés dans ces amplificateurs ultra intenses, constitue donc une nécessité absolue pour le développement des sources de demain et l'accès aux futures applications sous jacentes. En particulier, il est important dans un premier temps de lever l'incertitude au sujet du seuil de dommage des cristaux de Ti : Saphir. Afin de surmonter ce verrou, nous lançons ainsi une analyse de tous les facteurs qui influencent le seuil de dommage dans les cristaux de Ti:Sapphire dans le régime nanoseconde, picoseconde et femtoseconde [1, 2].

\section{PROPRIÉTÉS DU SAPHIR $\left(\mathrm{AL}_{2} \mathrm{O}_{3}\right)$}

Le saphir $\left(\mathrm{Al}_{2} \mathrm{O}_{3}\right)$ est un cristal très isolant uniaxe, de structure cristalline rhombohédrique, et caractérisé par un lien mixte ionique et covalent [3]. Il a un gap de grande énergie, et une concentration très basse de défauts. Il est chimiquement stable, dur, rigide et robuste mécaniquement et possède une très bonne conductivité thermique.

La nature ionique du saphir conditionne le fait que la bande de valence est dérivée des niveaux du $\mathrm{O} 2 \mathrm{p}$ et la bande de conduction dérive des niveaux du $\mathrm{Al} 3 \mathrm{~s}$ et $3 \mathrm{p}$. La structure des bandes peut être calculée avec la méthode PSF "pseudo-function band structure method", qui est une méthode basée sur les principes premiers. Cette méthode ne se base pas sur des données empiriques, mais simplement sur la position des atomes et leur nombre atomique. La PSF sous-estime légèrement la valeur du gap et ne calcule pas les effets "many-body". Le diagramme de bande du saphir calculé dans l'espace k est représenté ci-contre. Nous pouvons remarquer que le gap estimé est de $6,5 \mathrm{eV}$, alors que le gap expérimental estimé par des moyens optiques (lumière UV) est de $8,48 \mathrm{eV}$ avec une coupure de Urbach (exponentielle), typique des matériaux ioniques.

Plusieurs remarques découlent du schéma des niveaux des bandes :

a- le gap a une dépendance par rapport à la température,

$$
E_{g}(T)=E_{g}(T=0 K)+b T+g T^{2},
$$

Où $b$ est le facteur lineaire, et $g$ est le facteur quadratique du coefficient de température du gap, et $\mathrm{E}_{\mathrm{g}}(\mathrm{T}=0 \mathrm{~K})$ est la valeur du gap à 0 Kelvin.

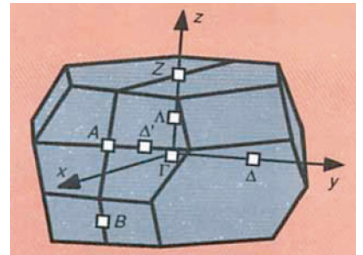

a)

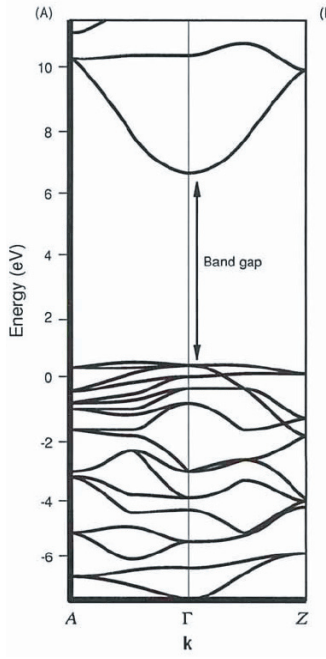

b)

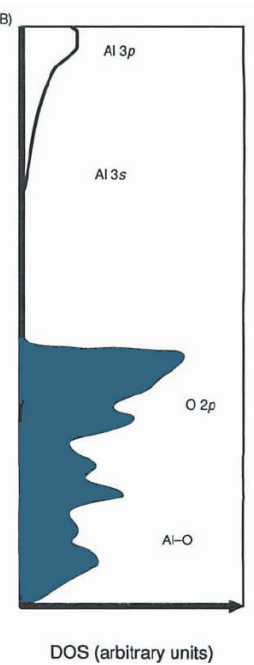

Figure 1. a) Espace réciproque: zones de Brillouin de la cellule unitaire réelle de a- $\mathrm{Al}_{2} \mathrm{O}_{3}$. b) Niveaux énergétiques et probabilité de saut pour 1 e $\mathrm{Al}_{2} \mathrm{O}_{3}$ à température ambiante (déterminés par la technique PSF). 
b- le décalage "Stokes" des niveaux de la bande de conduction est estimé à $1 \mathrm{eV}$, donc il y a un niveau de relaxation métastable au-dessous de la bande de valence.

\section{EXPÉRIENCES}

Nous avons mené deux types d'expériences : l'étude du seuil de dommage de la surface des échantillons et l'étude du seuil de dommage en volume (ou bulk) des échantillons.

Pour chaque expérience nous avons développé un protocole de détection des dommages adapté à l'étude réalisée.

Les caractéristiques principales du système laser $\mathrm{Ti}$ : Saphir amplifiée utilisé pour les expériences, Hurricane (Spectra-Physics), sont les suivantes : Energie $=400 \mu \mathrm{J}$, Durée temporelle $=$ $100 \mathrm{fs}$, Tauxderptition $=1 \mathrm{kHz}-100 \mathrm{~Hz}$, Diamètre du faisceau en champ proche: $6 \mathrm{~mm}$, Polarisation horizontale. Le paramètre $\mathrm{M}^{2}$ a été estimé par la mesure des dimensions du faisceau en trois plans de propagation du faisceau (proche, médian et lointain). Il est égal à : $\mathrm{M}^{2}=1,3$ selon les deux axes.

Les dommages ont été détectés avec un microscope, et définis comme tout "changement visible (par un microscope, par visualisation par une caméra et/ou par observation de la diffusion d'un laser $\mathrm{He} / \mathrm{Ne}$ ) des propriétés de l'échantillon (surface ou volume), induites par un rayon laser de puissance élevée" [2].

\section{1 Étude de l'endommagement dans le volume (bulk) du matériau}

\subsubsection{Montage expérimental}

Le dispositif expérimental est composé par les éléments suivants (Fig.2) :

- un système d'atténuation du faisceau constitué d'une lame $\lambda / 2$ et d'un polariseur;

- un puissance-mètre pour mesurer l'énergie sur cible. Le rapport énergie sur cible/énergie au point de mesure a été établi au début de l'expérience.

- une photodiode, alignée sur la perte d'un miroir, et calibrée auparavant, pour obtenir une mesure plus fine de la variation d'énergie ;

- un obturateur (shutter) rapide capable de sélectionner, à partir d'un train de $100 \mathrm{~Hz}$, une seule impulsion (temps de montée $3 \mathrm{~ms}$, temps de descente 7-8 ms).

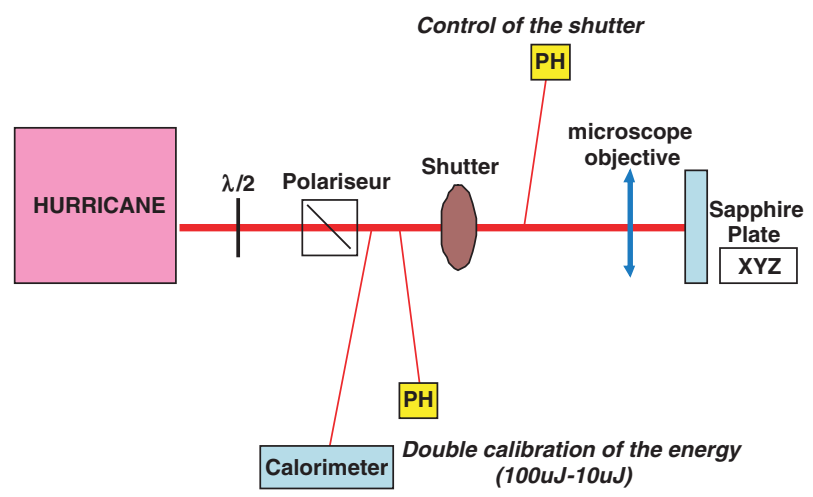

Figure 2. Dispositif expérimental des expériences pour déterminer le seuil de dommage dans le volume (bulk) du matériau. 
- une photodiode pour vérifier la synchronisation entre le déclenchement de l'obturateur et le train d'impulsions laser. On mesure ainsi l'énergie transmise pour vérifier que l'obturateur ne coupe pas une partie de l'impulsion.

- le focalisation sur la cible est réalisée par un objectif de microscope (objectif Zeiss LD-epiplan x 50 d'ouverture numérique $\mathrm{NA}=0,5$ et de grande distance de travail $(7 \mathrm{~mm})$ ).

- la cible est montée sur une translation XYZ pour garantir l'alignement et le tirage pour les expériences.

\subsubsection{Diagnostic pour la détection du dommage dans le volume (bulk) du matériau}

Le diagnostic des dommages est basé sur la détection des anneaux de diffusion d'un laser He-Ne colinéaire au faisceau à 800 nm (Fig.3.)

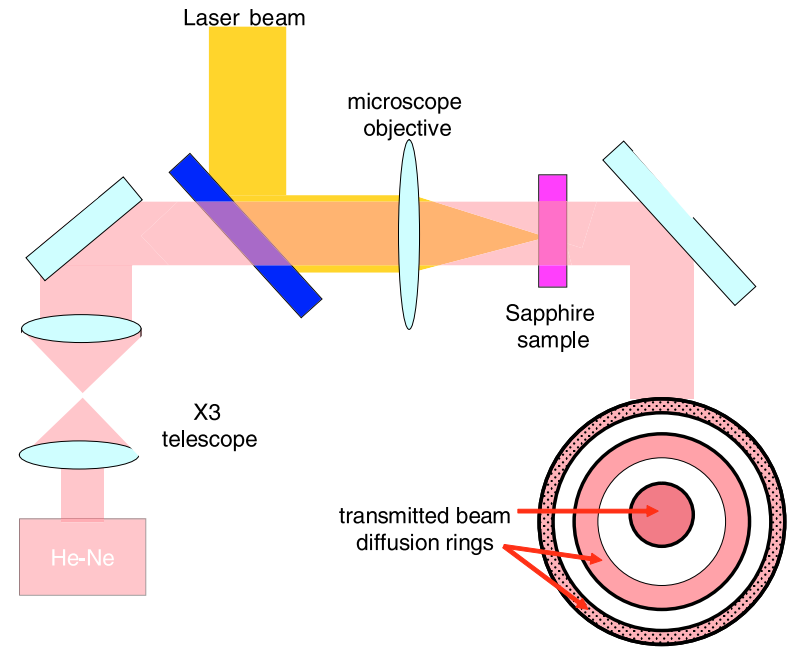

Figure 3. Diagnostic basé sur la détection de la diffusion d'un laser $\mathrm{He} / \mathrm{Ne}$.

\subsubsection{Résultats et commentaires}

Lors de cette expérience, nous avons étudié l'évolution du seuil de dommage en fonction du nombre de tir et du dopage du matériau. Les dopages étudiés sont « oscillateur », facteur $\alpha$ egal à trois, et « amplificateur », facteur $\alpha$ egal à un. Le facteur $\alpha$ est défini tel que l'adsorption $\mathrm{A}$ du cristal, à la longueur d'onde du laser de pompe $(532 \mathrm{~nm})$, est $\mathrm{A}=\exp (-\alpha \mathrm{l})$, avec 1 épaisseur du cristal.

Le seuil de dommage d'un matériel bulk transparent n'est pas très facile à mesurer directement. Dans les expériences l'estimation de la taille du spot focale est souvent affectée par les effets cumulés de l'auto-focalisation, de la dispersion et de l'auto-modulation de phase. Pour bien estimer le seuil de dommage dans le bulk d'un matériel transparent il faudra s'affranchir de tous ces effets. Le phénomène plus important reste l'auto-focalisation, car dans nos conditions d'expérience, avec des impulsions laser de $100 \mathrm{fs}$ de durée et avec des cibles de quelques $\mathrm{mm}$, la dispersion et l'auto-modulation de phase sont moins importantes. Pour contrôler cet effet nous avons augmenté la NA (ouverture numérique) du faisceau focalisant. Le critère à évaluer est la puissance critique car l'auto-focalisation est liée à cette valeur. La formules utiles à ce propos est celle qui permet de mesurer la puissance critique $\mathrm{P}_{c r}=$ $3.77 * \lambda^{2} / 8 * \pi \mathrm{qn}_{0} * \mathrm{n}_{2}$, avec $\lambda \mathrm{g} 800 \mathrm{~nm}, \mathrm{n}_{0}[$ Saphir $]=1.76, \mathrm{n}_{2}[$ Saphir $]=3.2 \cdot 10-16 \mathrm{~cm}^{2} / W$. Dans notre cas $\mathrm{P}_{c r}=1.7 \mathrm{MW}$. L'intensité d'endommagement $\left(10^{12}-10^{13} \mathrm{~W} / \mathrm{cm}^{2}\right)$ du saphir en regime fs est atteinte 
dans toutes les expériences sur des petites taches focales (avec un objectif de microscope par example) pour rester au-dessous de ce seuil et faire des estimations à partir de l'optique gaussienne. L'imprécision sur la mesure du seuil de dommage dans le volume (bulk) provient de l'estimation de la taille du waist car les autres effets non-linéaires, secondaires par rapport à l'auto-focalisation, comme la diffraction du plasma, réduisent la précision d'une estimation basée uniquement sur la propagation gaussienne du faisceau) dans le cristal.

Les résultats obtenus sont les suivants :

- Seuil de dommage vs nombre de tirs:

Les résultats de l'endommagement en volume (bulk) du saphir pur et monocristallin montrent une diminution du seuil avec l'exposition et suggèrent un effet d'incubation. Les résultats sont résumés dans le tableau 1.

- Seuil de dommage vs dopage:

Les trois différents dopages testés sont le saphir pur, le dopage oscillateur $(\alpha=1)$ et le dopage amplificateur $(\alpha=3)$.

Tableau 1. Variation du seuil avec le temps d'exposition (nombre de tirs) de l'échantillon pour le saphir pur (a) et seuil monocoup pour les échantillons dopage oscillateur et amplificateur (b).

\begin{tabular}{|c|c|}
\hline Bulk sapphire & $\mathrm{E}\left(\mathrm{J} / \mathrm{cm}^{2}\right)$ \\
\hline \hline SingleShot & $99 \pm 19$ \\
\hline $15 \mathrm{kSHOTS}$ & $79 \pm 15$ \\
\hline $70 \mathrm{kSHOT}$ & $39 \pm 8$ \\
\hline
\end{tabular}

\begin{tabular}{|c|c|}
\hline Bulk Ti :Sa & $\mathrm{E}\left(J / \mathrm{cm}^{2}\right)$ \\
\hline \hline dopage osc & $9 \pm 3$ \\
\hline dopage amp & $8 \pm 2$ \\
\hline
\end{tabular}

\section{2 Étude de l'endommagement en surface du matériau}

\subsubsection{Montage expérimental}

Le montage expérimental est similaire à celui utilisé pour les expériences dans le volume (bulk), sauf pour la zone d'interaction qui est sous vide $\left(10^{-6} \mathrm{mbar}\right)$, pour éviter la filamentation dans l'air (Fig.4) comme cela avait été constaté sous atmosphère d'air.

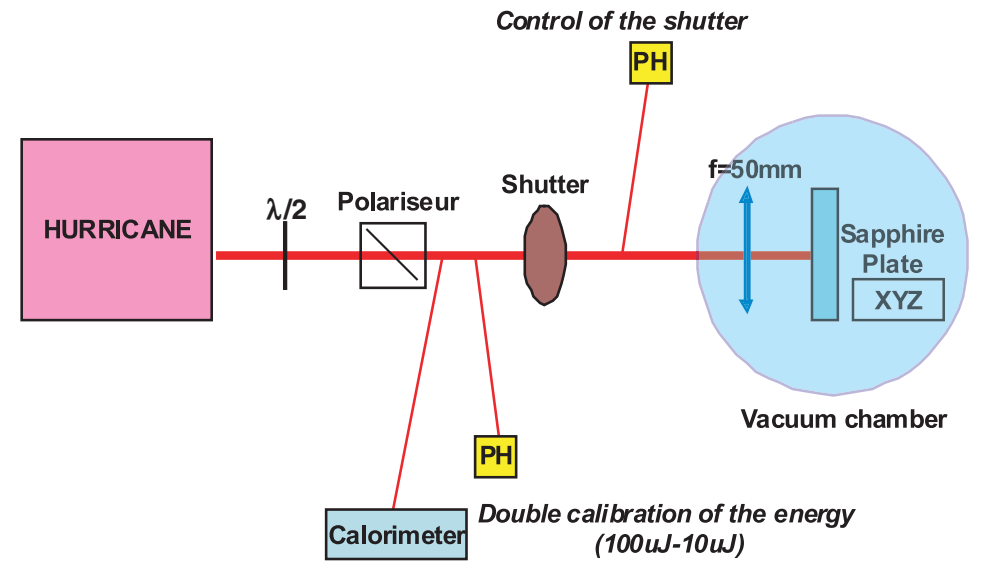

Figure 4. Dispositif expérimental des expériences en surface. 


\subsubsection{Diagnostic pour la détection du dommage en surface du matériau}

Le diagnostic pour la détection du dommage est composé d'une CCD et d'un système d'imagerie de la surface de la cible (Fig.5). Dans ce cas, tout défaut visible par ce système d'imagerie constitue le diagnostic d'endommagement.

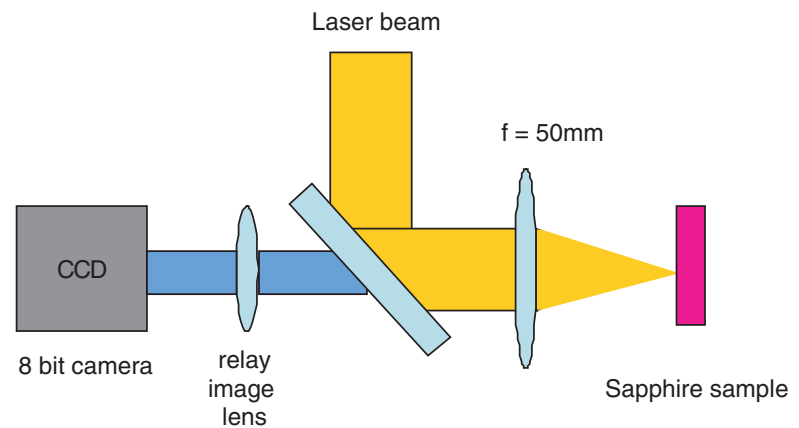

Figure 5. Diagnostic d'endommagement basé sur l'imagerie par CCD.

\subsubsection{Résultats}

Les résultats de l'endommagement de la surface du saphir pur et du dopage oscillateur $(\mathrm{a}=3)$ montrent une diminution du seuil avec le temps d'exposition (nombre de tirs) et suggèrent un effet d'incubation. Les résultats sont résumés dans le tableau 2.

Tableau 2. Variation du seuil avec le temps d'exposition de l'échantillon, pour le saphir pur (a) et dopage oscillateur (b).

\begin{tabular}{|c|c|}
\hline superpolish & $\mathrm{E}\left(\mathrm{J} / \mathrm{cm}^{2}\right)$ \\
\hline \hline $1 \mathrm{~ms}$ & $7.0 \pm$ \\
\hline $100 \mathrm{~ms}$ & $6.0 \pm$ \\
\hline $500 \mathrm{~ms}$ & $1.7 \pm$ \\
\hline $1 \mathrm{~s}$ & $1.3 \pm$ \\
\hline $10 \mathrm{~s}$ & $1.0 \pm$ \\
\hline
\end{tabular}

a)

\begin{tabular}{|c|c|}
\hline $\mathrm{Ti}: \mathrm{Sa}$ osc & $\mathrm{E}\left(\mathrm{J} / \mathrm{cm}^{2}\right)$ \\
\hline \hline $1 \mathrm{~ms}$ & $3.0 \pm$ \\
\hline $1 s$ & $0.8 \pm$ \\
\hline
\end{tabular}

b)

\section{CONCLUSIONS ET PERSPECTIVES}

Les premières expériences d'endommagement du saphir et Ti :Sa en regime $f s$ ont permis de chiffrer de manière quantitative le seuil de dommage de ces matériaux en fonction des paramètres laser incident classiques les des lasers de très haute intensité et pour différentes conditions préparatoires des matériaux. On a pu ainsi constater une grande dépendance du seuil de dommage en fonction du nombre de tirs (mise en évidence d'un phénomène d'incubation) et du dopage. La surface, que l'on peut apparenter à un défaut, est un élément également fortement limitant pour la résistance à l'endommagement puisque ce dernier est observé un facteur 10 en-deçà (typiquement $1-10 \mathrm{~J} / \mathrm{cm}^{2}$ selon la préparation (polissage, dopage) du matériau) par rapport aux valeurs mesurées en volume ((typiquement $10-100 \mathrm{~J} / \mathrm{cm}^{2}$ selon la préparation (polissage, dopage) du matériau). 
Les expériences futures prévoient une étude de l'influence des différents paramètres en régime d'interaction ps et ns, et en fonction des paramètres de préparation du matériau:

- polissage (produits, poudres, liquides),

- traitement AR (différents matériaux: $\mathrm{HfO}_{2}, \mathrm{Ta}_{2} \mathrm{O}_{5}, \mathrm{TiO}_{2}, \mathrm{ZrO}_{2}$ )

- dopage et inclusions (réseau cristallin inhomogène).

Une étude théorique des mécanismes d'endommagement sera aussi initiée pour mieux comprendre les phénomènes mis en jeu, notamment dans le cas d'un fonctionnement en régime répétitif (analyse du phénomène d'incubation) et optimiser la recherche des solutions à ce problème.

\section{Références}

[1] F. Canova, J.-P. Chambaret, G. Mourou, M. Sentis, O. Uteza, P. Delaporte, T. Itina, J.-Y. Natoli, M. Commandre, C. Amra, Complete characterization of damage threshold in titanium doped sapphire crystals with nanosecond, picosecond and femtosecond laser pulses, Boulder Damage Symposium 2005

[2] Normes ISO Lasers et équipements associés aux lasers - Détermination du seuil d'endommagement provoqué par laser sur les surfaces optiques : 11254-1 et 11254-2

[3] R. H. French, Electronic Band Structure of $\mathrm{AI}_{2} \mathrm{O}_{3}$, with Comparison to AlON and AIN, J. Am. Ceram. Soc., 13 (1990) 471-489 